\title{
Reducing Waste in the Critical Care Setting
}

Jean Morrow, RN, BScN

Clinical Educator, Critical and Neurosurgical Care

Shelia Hunt, RN BScN

Medical-Surgical Intensive Care Unit

Virginia Rogan, RN

Kathryn Cowie, RN

Jan Kopacz, RN

Medical-Surgical Intensive Care Unit

Colleen Keeler

Manager, Quality and Patient Safety

Mary Beth Billick, RN, BScN

Coordinator, Medical-Surgical Intensive Care Unit

Mary Kroh, RN, BScN

Coordinator, Cardiac Surgery Recovery Unit

London Health Sciences Centre - University Hospital

\begin{abstract}
Background: The ICU at London Health Sciences Centre - University Hospital (LHSC-UH) is a 40-bed critical care unit that contains two separate supply rooms that carry all the essential materials necessary for patient care. However, considering the patient acuity in critical care, it is vital that this equipment is made more accessible for practitioners at the bedside. Therefore, nurse servers or bedside supply cabinets are present in each of the patient rooms. While these servers provide timely access to the supplies essential for nursing care, they are also a huge source of waste. When patients who are identified as having antibiotic-resistant organisms (AROs) are discharged, numerous unused items are discarded for infection control purposes.
\end{abstract}


Aims and objectives: Project objectives were to curtail waste by minimizing stocked supplies at the bedside, exploring alternative stocking options and increasing awareness of this issue with practitioners.

Methods: An interprofessional team was formed consisting of registered nurses, support service workers, environmental service workers, infection control practitioners and critical care leadership. A cost analysis of discarded supplies was undertaken, and results were communicated to all staff. Infection control practitioners developed guidelines specific to use of the nurse servers and linen supply areas. The stocking process and contents of the servers were reviewed; surplus was removed and relocated to a close central area outside patient rooms. Following agreement on new server contents, lists and photos were created and posted in each supply room. New stocking guidelines were phased in gradually and were adapted according to user feedback.

Results: Over a two-week period, a pilot cost analysis identified that supplies valued at $\$ 2,327.25$ had been discarded from five bedsides. Future long-term cost savings will enable management to redirect such resources and therefore improve other essential care services in the ICU.

Conclusion: Increasing awareness of wasteful stocking practices facilitated the engagement of this CQI project. New stocking practices have greatly reduced waste and increased service efficiencies while maintaining the integrity of optimal patient care.

\section{Introduction}

In an ongoing effort to provide best patient care, nurses are challenged with both maintaining an environment that is safe for all patients, while at the same time being responsible stewards of the public healthcare system. The obstacles that nurses face in sustaining this delicate balance include mitigating expenses, minimizing waste and adhering to infection control guidelines. In particular, the use of nurse servers in patient rooms provides easy access to supplies that are commonly required to facilitate patient monitoring and provision of highquality care. Nurse servers in the ICU are bedside wheeled cabinets that are regularly stocked with important medical and personal care supplies such as gauze, needles, syringes, blood tubes, mouth swabs, IV caps, occlusive dressings and linens. They provide timely and convenient access to vital resources.

Antibiotic-resistant organisms (AROs) are bacteria that are resistant to traditional antibiotic therapy. In fact, one study reports that as much as $10 \%$ to $20 \%$ of bacteria cultured in hospitals are resistant to commonly used antibiotics 
(CARA 2006). The increased incidence of AROs in patient care settings creates additional expenses, requires complicated treatment and lengthens hospital stays (NIH 2013). Ever present in the healthcare setting, especially in susceptible patient populations, these organisms require stringent infection control protocols. One such measure involves discarding all disposable supplies from an ARO patient room upon the patient's transfer or discharge. In the ICU, patients are at increased risk of acquiring and transmitting these infections owing to critical illness and immunosuppression. In this regard, nurse servers present a dichotomous predicament: they provide convenient access to supplies but also the potential contamination and wastage of stocked products. Owing to the unpredictable nature of patient care in the ICU, and the fact that patient outcomes are directly correlated with timely intervention, it is essential that staff have easy access to principal medical supplies. Unfortunately, clean supply rooms are not always conveniently located, and patient care is delayed as a result. Alternatively, if unused items in bedside nurse servers become ARO contaminated, this poses an additional health and safety risk to patients.

In 2012, the combined Medical-Surgical ICU (MSICU) and Cardiac Surgery Recovery Unit (CSRU) at LHSC-UH initiated a continuous quality improvement (CQI) project aimed at reducing waste generated by patients with AROs who are discharged or transferred from ICU. The ICU waste reduction initiative aligns well with LHSC's corporate call to action regarding patient safety and infection control. Specifically, LHSC's "Call to Action: Infection Safety" identifies the following desirable outcomes: (a) reduced rates of infection and transmission, (b) organizational culture change regarding ARO prevention and management, (c) developing sustainable strategies and (d) fostering a network of highly committed stakeholders through multidisciplinary engagement (LHSC 2012).

The CSRU and MSICU have a combined 39-bed capacity and a shared staff of 230 nurses; they treat approximately 5,120 patients annually. Core interdisciplinary CQI group members included nursing coordinators, registered nurses (RNs), support service workers (SSWs), environmental service workers (ESWs) and an infection control consultant. Charged with the task of investigating the current extent of cost and wastage associated with oversupply of the nurse servers, this group generated stocking and waste process recommendations. These recommendations included strategies aimed at reducing waste and cost expenditures, improving infection control measures and highlighting environmental issues, while still maintaining optimal patient care and safety. Moreover, the CQI team was encouraged to forge positive solutions that not only enacted 
change within their own unit but that also provided a catalyst for practice change across the hospital.

\section{Background}

A literature review was undertaken looking at the major concept of waste reduction and its relationship to expected outcomes, including environmental protection, cost savings and improved infection control. Reducing the amount of discarded unused medical supplies in the ICU is believed to reduce organizational costs, help protect the environment and reduce infectious transmission of AROs. Both hospitals and healthcare institutions produce a significant amount of waste. In fact, in 2007 American healthcare facilities were estimated to produce 13,200 pounds of waste every single day (Lauer 2009). This waste is a large part of their environmental footprint (Daschner and Dettenkofer 1997; Lauer 2009). In an effort to reduce expenditures and become more environmentally friendly, institutions have sought out cost-saving solutions for reducing waste in their facilities. A review of current literature highlighted a variety of initiatives that have been developed to reduce waste, including systems reviews, practice changes and environmental campaigns. For example, the diversion of non-hazardous waste from medical waste is one initiative that has received much attention. A 2011 report from the Archives of Surgery indicated that up to $90 \%$ of waste entering the hazardous stream did not belong there (Kwakye et al. 2011). Furthermore, the researchers estimated that a significant savings could be achieved if waste was entered into the appropriate stream, considering that $86 \%$ of waste disposal costs derive from hazardous medical waste materials (Kwakye et al. 2011).

There is a growing body of literature on waste analysis and waste reduction in the context of operating rooms (ORs). Several sources report possible changes that should be considered to reduce waste in these areas, such as in the orthopaedic surgery division (Lee and Mears 2012). While only one study highlighted waste reduction in critical care, the initiative Greening Critical Care did not specifically address the removal of unused supplies from ARO environments. The authors identified hospital waste as a major contributor to landfills (Chapman and Chapman 2011).

Nurses and, indeed, all healthcare professionals should be environmentally aware, recognizing that needless hospital waste, including the incineration of biohazardous materials, has a devastating effect on our environment. Environmental pollution is a significant byproduct of medical waste incineration (Daschner and Dettenkofer 1997). Various hospitals have developed 
environmental awareness campaigns that encourage staff to use medical and personal care supplies judiciously, and they have provided clear instructions regarding the disposal of these materials (Swartz 1012). Other hospitals have adopted the "go green" motto of collective environmental responsibility by encouraging staff to follow the three Rs (reduce, reuse and recycle). However, the literature does not provide any information regarding specific wastage of potentially ARO-contaminated material (e.g., unused medical and personal bedside supplies).

In addition to the environmental benefits, there are significant cost savings related to the process of waste reduction. Discussion in the literature regarding the costs associated with AROs has traditionally focused on laboratory, screening, treatment, hospitalization and staffing outcomes (Kim et al. 2001). However, in Canadian hospitals, the economic impact of MRSA alone is estimated to be \$33-42 million (Health Canada 2002). To date, waste management plans have proven highly effective, with some organizations reporting as much as a $58 \%$ reduction in medical waste and nearly $50 \%$ in cost savings (Almuneef and Memish 2003).

Patients with AROs are placed in isolation to prevent the spread of disease. Everything in the ARO patient environment needs to be properly cleaned or disposed of once the patient is deceased or discharged from the room in order to prevent cross-contamination of other patients and staff. While the literature demonstrates a debate between using disposable versus reusable supplies with ARO-positive patients, what remains constant is the practice of ensuring that any item that has been in contact with the patient "should not come into either direct or indirect contact with other patients" (Bagshawe et al. 1978: 810). It is clear that waste costs have risen in recent years as a direct result of implementing tighter infection control measures that are intended to protect patients from ARO infections. Conversely, there is no evidence that waste reduction adversely affects infection rates, especially where practice changes are carefully executed to protect patients, as was the case in our ICU waste reduction initiative.

\section{Design and Implementation}

Practice change is highly dependent on staff engagement (Ferenc 2010). This project was dedicated to promoting involvement at every level. It started with the ICU leadership team, which recruited the original interprofessional Waste Reduction Working Group that began this project. The working group included registered nurses, infection control practitioners, SSWs, ESWs, a quality and patient safety expert, an inventory control clerk and leaders from both criti- 
cal care and support services. From the onset leadership supported the group's ideas, provided necessary resources and encouraged their efforts towards environmental stewardship, improved infection control and cost reduction.

After an initial meeting, the group met biweekly to brainstorm, plan and evaluate the objectives. Meetings were held in the traditional setting of a conference room, but also consisted of walking through ICU patient care areas. Informal walkabouts facilitated visualization of problem areas and provided an open venue for innovative solutions. Interest from front-line staff grew as they were asked to contribute their thoughts and ideas as well as take part in piloting several waste reduction strategies.

The group reviewed infection control guidelines for the safe handling of supplies after discharge of patients with AROs. These guidelines were offered in order to minimize ARO transmission and to reduce unnecessary wastage. With new infection control guidelines in place, a collective decision was made to perform a cost analysis based on the collection of discarded supplies from five bedsides over a two-week period. A total of $\$ 2,327$ worth of discarded unused items were collected and inventoried during this time. Furthermore, based on size and equal patient acuity, it was determined that both MSICU and CSRU could generate similar savings. In light of the potential cost savings, it became clear that devising a long-term strategy for waste reduction was required to achieve and sustain meaningful change.

Information was provided to staff via email, including photographs, posters, a hospital newsletter and a trolley with the wasted supplies, which were displayed for staff to view for themselves the current state of wastage. It is believed that these efforts facilitated staff reflection and helped them embrace the need for practice change. According to Almuneef and Memish (2003), allowing staff to identify their own needs and then actively engaging them in finding the solutions enhances learning and the adoption of a new practice.

While waste and cost reduction were at the forefront of the project, so was practitioner efficiency. To enhance staff buy-in, a storage area close to the patient room was needed to house the removed supplies. Therefore, supply "boats" were created to contain displaced supplies in common areas. These boats contained commonly used personal care supplies such as mouth swabs.

Once the stocking process itself was agreed upon, a discussion regarding implementation was started. The team contemplated whether the changes should be rolled out gradually and piloted in a smaller area, or whether a department-wide 
approach was a better option. A gradual transition, starting with two bedsides, was decided upon. The implementation process was slow; however, it proved more effective because it allowed for staff reflection and feedback, which further engaged them in the project. Staff were all offered educational inservices and were sent emails outlining the changes; they viewed posters and participated in hands-on training, all methods designed to improve project success (Almuneef and Memish 2003). Solicitation of feedback was active throughout the trial and was used to evaluate the changes prior to unit-wide rollout.

\section{Objectives}

The ICU waste reduction project sought to curtail waste through the following initiatives: (a) minimizing supplies stocked on nurse servers, (b) exploring alternative stocking options and developing solutions, (c) considering environmental implications and (d) increasing direct care providers' awareness of cost, waste and infection control issues.

\section{Positive Outcomes}

Now that new stocking guidelines for servers have been fully implemented, nurses have assumed responsibility for ensuring that they have planned ahead and are anticipating supply needs prior to entering patient rooms. New stocking practices have led directly to significant cost savings, allowing funds to be redirected to other patient care services. Additionally, fewer unopened supplies end up in local landfill, a positive contribution to environmental protection.

As a result of the collaboration required to enact this initiative, we have experienced improved teamwork between nurses and SSWs. Specifically, nurses will now alert the SSWs to stock the carts according to patient condition. For example, if the patient is slated for discharge in the next 24-48 hours, the nurse advises the SSW that general restocking may not be required and provides a short list of essential items needed. This process further reduces overstocking and eventual wastage. Consequently, the enhanced communication between team members has fostered an appreciation and understanding of each other's role in patient care.

Overall ARO infection rates in the unit have declined. Although we need to examine the correlation between waste reduction and ARO infection rates in the ICU, it is anticipated that the waste reduction initiative has contributed to its decline. The decrease in ARO transmission may be related to (a) a reduction of contaminated supplies, (b) fewer trips into ARO-contaminated rooms to restock and (c) decreasing the amount of contaminated waste leaving the room. 


\section{Challenges and Recommendations}

Although there were several successes, some challenges arose. Primary challenges included initial staff resistance, finding immediate solutions for relocated supplies and staff education. Encouraging staff to accept this practice change entailed a great deal of discussion and dialogue. It is recognized that critical care patients change rapidly, and while some situations can be anticipated, others cannot. Nurses felt the reduced availability of supplies on nurse servers created a dangerous patient care environment. They voiced the concern that keeping supplies outside ARO-contaminated rooms required additional donning and doffing of personal protective equipment. Also, they felt that time spent collecting supplies ultimately delayed treatment and therefore jeopardized patient safety. Ongoing staff education was necessary to clarify the rationale for reduced supplies and to discuss strategies that would mitigate any negative effects on patient care. Such dialogue was helpful in overcoming these challenges.

Finding immediate storage for the supplies removed from nurse servers was the second challenge. Because of unit design and patient acuity, central supply areas are challenging for staff to access readily. This situation prompted a solution that allowed displaced supplies to remain readily accessible and close to the patient. As a result, small additional storage boats were created and positioned outside patient rooms.

The final challenge was to ensure ongoing education and communication with staff in order to support and sustain the practice changes. A multifaceted educational plan was implemented; written communications included visual aids such as photos, supply template lists and hands-on practice. In addition, breaking through existing work culture was a difficult hurdle to overcome. Ongoing support was required to shift staff's attitudes and practices. CQI team members championed this initiative every day in their own practice, encouraging and supporting colleagues to embrace the changes through highlighting the benefits to both staff and patients. They also provided one-on-one communication, email reminders and other forms of correspondence that met individual staff's learning needs.

Recommendations for practitioners undertaking similar future initiatives:

1. Include front-line staff throughout the process.

2. Be expert communicators through a variety of media (email, posters, face-to-face conversations).

3. Consider complete implementation on rollout rather than staggered implementation. 


\section{Conclusion}

Staff members are effectively adjusting to the practice changes resulting from this initiative. The objective of reducing waste from ARO-positive rooms has been achieved, and with that, cost savings have been realized. We know there have been savings, although no credible data exists to support a specific number. Moreover, the reduction in waste helps to promote an environmental awareness that is more consistent with Canadian values and helps alleviate some of the increasing demand on our waste management systems. Additionally, infection control practices have remained consistent, and there is significantly less ARO-contaminated waste leaving patient care areas.

In the past, staff voiced that there were only limited opportunities for them to have meaningful impact on their work environment. This CQI project provided a real opportunity for staff members to become engaged, drive the project forward and achieve a positive impact at both the micro and macro levels. Individual empowerment enabled staff to take charge of their own practice while feeling they were contributing to greater efforts towards infection control and waste management. Outcomes affected all interprofessional practitioners including leadership, nursing staff and allied healthcare providers; adding a sense of pride and accomplishment that was palpable on the front line.

In the words of Archbishop Desmond Tutu: "Do your little bit of good where you are; it's those little bits of good put together that overwhelm the world" (Sustainable Baby Steps 2013).

\section{Acknowledgements}

We would like to acknowledge the timely and efficient support of Minakshi Sharma, the Clinical Librarian at London Health Sciences Centre, who performed the literature search for this paper. We would also like to thank Lisa Ducharme, of the Nursing Professional Practice group, for her support in reviewing, editing and championing this paper. Additionally, our thanks go to the Waste Reduction Working Group, whose members made this project their own and were instrumental in its success. 
Correspondence may be directed to: Jean Morrow, Clinical Educator: Critical Care and Neurosurgery, London Health Sciences Centre, University Hospital, 339 Windermere Road, PO Box 5339, London, Ontario, N6A 5A5; e-mail: Jean.morrow@lhsc.on.ca

\section{References}

Almuneef, M. and Z.A. Memish. 2003. "Effective Medical Waste Management: It Can Be Done." American Journal of Infection Control 31(3): 188-92.

Bagshawe, K.D., R. Blowers and O.M. Lidwell. 1978. "Isolating Patients in Hospital to Control Infection. Part IV: Nursing Procedures.” British Medical Journal 2(6140): 808-11.

Canadian Antimicrobial Resistance Alliance (CARA). 2006. "Comprehensive Overview of Antibiotic Resistance in Canada.” Retrieved March 26, 2013. <http://www.can-r.com/mediaResources/ComprehensiveOverview.pdf $>$.

Chapman, M. and A. Chapman. 2011. “Greening Critical Care.” Critical Care 15(2): 302. Retrieved March 25, 2013. <http://www.ncbi.nlm.nih.gov/pmc/articles/PMC3219402/pdf/cc9409.pdf>.

Daschner, F.D. and M. Dettenkofer. 1997. "Protecting the Patient and the Environment: New Aspects and Challenges in Hospital Infection Control." Journal of Hospital Infection 36(1): 7-15.

Ferenc, J. 2010. “Going Greener. Hospitals Continue to Make Sustainability a Priority.” Health Facilities Management 23(12): 24-28.

Health Canada. 2002. "Prevention and Control of Occupational Infections in Health Care. An Infection Control Guideline." Canada Communicable Disease Report (CCDR) 28(1): 1-264.

Kim, T., P.I. Oh and A.E. Simor. 2001. “The Economic Impact of Methicillin-Resistant Staphylococcus aureus in Canadian Hospitals." Infection Control and Hospital Epidemiology 22(2): 99-104.

Kwakye, G., G.A. Brat and M.A. Makary. 2011. “Green Surgical Practices for Health Care.” Archives of Surgery 146(2): 131-36. doi: 10.1001/archsurg.2010.343.

Lauer, M. 2009. “Reducing Health Care's Ecological Footprint.” American Journal of Nursing 109(2): 56-58. doi: 10.1097/01.NAJ.0000345439.68228.a0.

Lee, R. and S. Mears. 2012. "Greening of Orthopedic Surgery." Orthopedics 35(6): 482.

London Health Sciences Centre (LHSC). 2012. "Call to Action: Infection Safety." Retrieved March 26, 2013. <http://www.lhsc.on.ca/priv/csi/index.html>.

National Institutes of Health (NIH). 2013. Antimicrobial (Drug) Resistance. Retrieved March 26, 2013. <http://www.niaid.nih.gov/topics/antimicrobialresistance/Pages/default.aspx>.

Sustainable Baby Steps. 2013. Environment Quotes. Retrieved March 26, 2013. <http://www. sustainablebabysteps.com/environment-quotes.html>.

Swartz, T. 2012. “Going Green and Lowering Costs with IC in Mind.” Patient Safety Monitor Journal 13(5): 6-8. 